\title{
Immunological evaluation of chitosan nanoparticles loaded with tetanus toxoid
}

\author{
Ghalavand $\mathrm{M}^{1}$, Saadati $\mathrm{M}^{2}$, Ahmadi $\mathrm{A}^{3}$, Abbasi $\mathrm{E}^{4}$, Salimian $\mathrm{J}^{5}$ \\ Chemical Injuries Research Center, System Biology and Poisonings Institute, Baqiyatallah University \\ of Medical Science, Tehran, Iran. jafar.salimian@gmail.com
}

\section{ABSTRACT}

OBJECTIVES: The present study was aimed at comparing tetanus toxoid (TT)-loaded-chitosan nanoparticles with aluminum hydroxide as a common vaccine adjuvant.

BACKGROUND: Tetanus remains to be a major public health problem. Nanoparticles have been extensively used as immune adjuvants. Tetanus toxoid (TT) encapsulated in chitosan nanoparticles is considered to be a promising tetanus vaccine candidate.

METHODS: TT-loaded chitosan nanoparticles were prepared by the ionic gelation method. The nanoparticles were studied by SEM for their size and morphology. In vivo study was conducted to evaluate the immunity response using mice divided into 4 groups and injected with encapsulated toxoid. The immune responses were then measured using indirect ELISA.

RESULTS: The purity and integrity of antigen were confirmed by SDS-PAGE electrophoresis. The size of nanoparticles was estimated at $100 \mathrm{~nm}$. As a result, the IgG antibody levels were 1.9, 1.76, and 0.87 in chitosan nanoparticles, aluminum hydroxide, and TT alone groups, respectively. Also, the immune responses were significantly higher in immunized groups compared to control groups vaccinated with free adjuvant vaccines $(p<0.05)$. CONCLUSIONS: The quality and efficacy of toxoid-loaded chitosan nanoparticles were reasonable. It enhanced the immune responses as much as aluminum hydroxide adjuvant does and thus may be a good alternative candidate (Tab. 1, Fig. 3, Ref. 16). Text in PDF www.elis.sk.

KEY WORDS: immunogenicity, nanoparticle, tetanus, chitosan.

\section{Introduction}

Tetanus is a medically important toxin causing high mortality especially in developing countries. Clostridium tetani, the causative agent, is commonly found in feces of domestic animals, humans and also in soil. Being responsible for tetanus clinical presentations, tetanus toxin is composed of two light and heavy chains interconnected with a disulphide bond (1). Fortunately, the incidence of the disease has dramatically decreased due to widespread vaccination. The vaccine is based on inactivated tetanus toxin (tetanus toxoid; TT) available as a single antigen or in combination with DTP vaccine (2). Adjuvants are added to vaccines to improve the vaccine-derived immunogenicity. Aluminum hydroxide as a mineral component adjuvant is widely used with high efficacy that induces early, high-titer, and long-lasting protective immunity (3). Its mechanism of action is through slow releasing of the antigen at

${ }^{1}$ Virology Research Center, Baqiyatallah University of Medical Science, Tehran, Iran, ${ }^{2}$ Biological Research Center, Faculty of Basic Sciences, Imam Hossein University, Tehran, Iran, ${ }^{3}$ Molecular Biology Research Center, Systems Biology and Poisonings Center, Baqiyatallah University of Medical Sciences, Tehran, Iran. ${ }^{4}$ Razi Vaccine and Serum Research Institute, Karaj, Iran, and ${ }^{5} \mathrm{Chemical}$ Injuries Research Center, Baqiyatallah University of Medical Science, Tehran, Iran

Address for correspondence: J. Salimian, Chemical Injuries Research Center, System Biology and Poisonings Institute, Baqiyatallah University of Medical Science, Tehran, Iran. Phone: 982182482503 , Fax: 982188211524 the injection site, activating complements and dendritic cells and inducing chemokines $(4,5)$. Chitosan, a deacetylated derivative of chitin is known as an excellent natural polymer with biocompatibility, biodegradability, non-toxicity, and adsorption properties. Chitosan has appropriate antibacterial, antifungal, and antiinflammatory properties that can be used as an effective adjuvant for immunization against tetanus (6). According to the importance and applications of polymeric nanoparticles in the field of vaccine development, the present study was conducted to synthesize tetanus toxoid-loaded chitosan nanoparticles (NPs) and to compare their induced immunity in mouse model with aluminum hydroxide.

\section{Material and methods}

\section{Antigen preparation and Protein assay}

Tetanus toxoid was obtained from Razi Vaccine and Serum Research Institute (Karaj, Iran). Protein concentration was measured using Bradford assay (7). In order to assess protein purity, SDSPAGE electrophoresis (12\%) was carried and Coomassi brilliant blue staining was used to visualize the protein.

\section{Nanoparticle preparation and evaluation}

Chitosan NPs were synthesized by ionic gelation method (8). Firstly, the chitosan solution (w/v $1 \%$ ) was prepared by dissolving chitosan powder (Sigma, USA) in acetic acid (1\%) and then sufficient amount of the antigen was added. Then, sodium tripolyphosphate $(10 \mathrm{mg} / \mathrm{ml})$ (Sigma, USA) was gradually added 
$71-74$

to the chitosan solution. Finally, the suspension was centrifuged and the supernatant was discarded, and the encapsulation rate was measured.

\section{Characterization of chitosan nanoparticles}

Scanning Electron Microscope (KYKY-EM3200, China) was used to investigate the morphological characteristics and distribution of the synthesized nanoparticles.

\section{Immunoassay}

Twenty female Swiss Webster mice were used for the purpose of being immunized with chitosan nanoparticles. The mice were divided into 4 groups $(n=5)$ and immunized with subcutaneous injections of tetanus toxoid as follows: Groups I, II, III and IV were immunized with tetanus toxoid and aluminum hydroxide as an adjuvant, tetanus toxoid and chitosan nanoparticles as an adjuvant, antigen alone, and empty nanoparticles, respectively. The dosages and injection intervals were the same for all groups. Tables 1 and 2 demonstrate time of administration, intervals, and dosages of injections. Also, $20 \mu \mathrm{g}$ antigen was used for the first injections. Subsequently, the booster injections were injected as 20,10 and 8 $\mu \mathrm{g}$ at 20 -day intervals between the first and second injections, and 14-day interval between the third and fourth injections.

Twenty days after the final injection, blood samples were taken from the retroorbital sinus. For indirect ELISA, briefly, $2 \mu \mathrm{g} / \mathrm{well}$ of the antigen was coated in the ELISA plate. After blocking ( $2 \% \mathrm{BSA})$, the sera were serially diluted two-fold from $1: 100$ to $1: 12800$ in PBS and peroxidase-labelled anti-mouse IgG antibody was added. Finally, the plate was read by means of ELISAPlate Reader at $450 \mathrm{~nm}$.

\section{Data analysis}

Means \pm standard deviations (SD) of three independent experiments were performed. The statistical analyses were carried

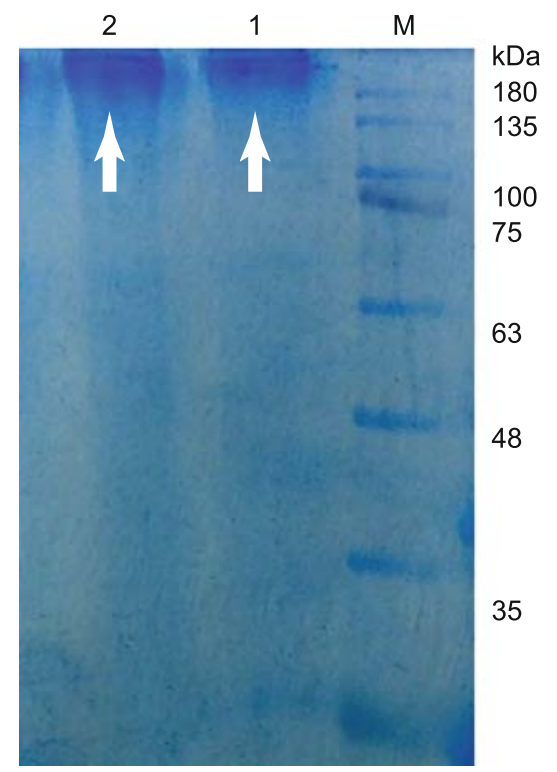

Fig. 1. SDS-PAGE of the antigen protein. M: Molecular marker; lanes 1 and 2: Antigen sample (tetanus toxoid).

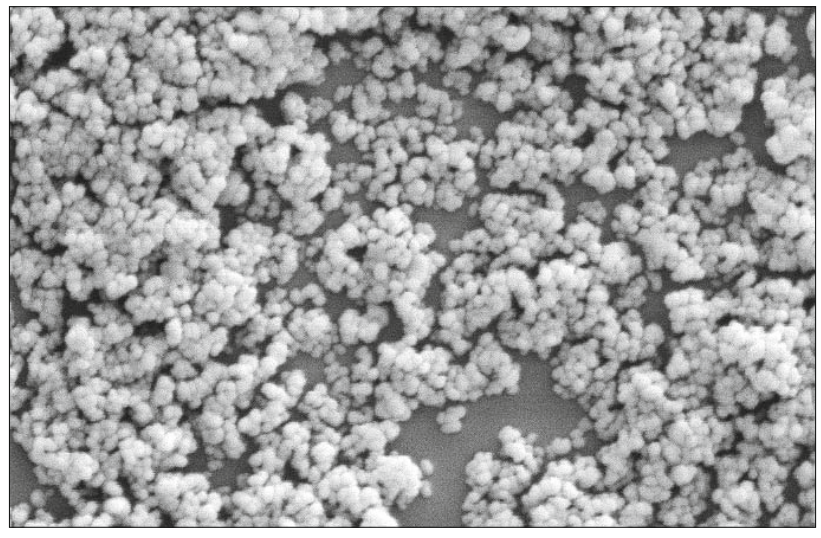

Fig. 2. Chitosan nanospheres encapsulating the protein antigen; nanospheres were fixed on a layered aluminum foil and imaged by SEM.

out with SPSS statistical software version 20. The obtained data were analyzed by Graphpad Insta software using one-way ANOVA (analysis of variances) and Tukey (post hoc) test. $p$ value $<0.05$ was considered significant.

\section{Result}

\section{SDS-PAGE}

SDS-PAGE electrophoresis (12\%) was performed to assess the purity of protein. The results showed purified protein concentrated by formaldehyde as a single dense band at the top of the gel (Fig. 1), confirming that no aggregate or fragment has occurred during entrapment.

\section{Nanoparticles scanning electron microscopy}

SEM imaging showed that the morphological features and distribution of nanoparticles were normal. Also, therein, the average size of the nanoparticles was around $100 \mathrm{~nm}$ and the encapsulation rate was estimated at $76 \%$ (Fig. 2).

\section{ELISA}

After injections, the antibody titers were measured in four mice groups using indirect ELISA immunoassay. The highest antibody level in group I (antigen + chitosan nanoparticle) was 1.9. There was a significant difference between group I and control group ( $p$ $<0.05)$. The highest level of antibody in group II (TT + aluminum hydroxide) was 1.79 . Also, there was a significant difference between group II and control group $(p<0.05)$. However, there was not any significant difference between groups I and II in terms of antibody titration (NS) (Tab. 1, Fig. 3).

Tab. 1. Statistical data's.

\begin{tabular}{cccccc}
\hline Groups & Components & No & Mean & SD & ST error \\
\hline 1 & TT + chitosan NP & 5 & 1.906 & 0.1647 & 0.07366 \\
2 & TT + alum adjuvant & 5 & 1.79 & 0.1597 & 0.07141 \\
3 & TT + PBS & 5 & 0.874 & 0.02302 & 0.0103 \\
4 & Np + PBS & 5 & 0.075 & 0.005244 & 0.002345 \\
5 & Control & 5 & 0.05 & 0 & 0 \\
\hline
\end{tabular}




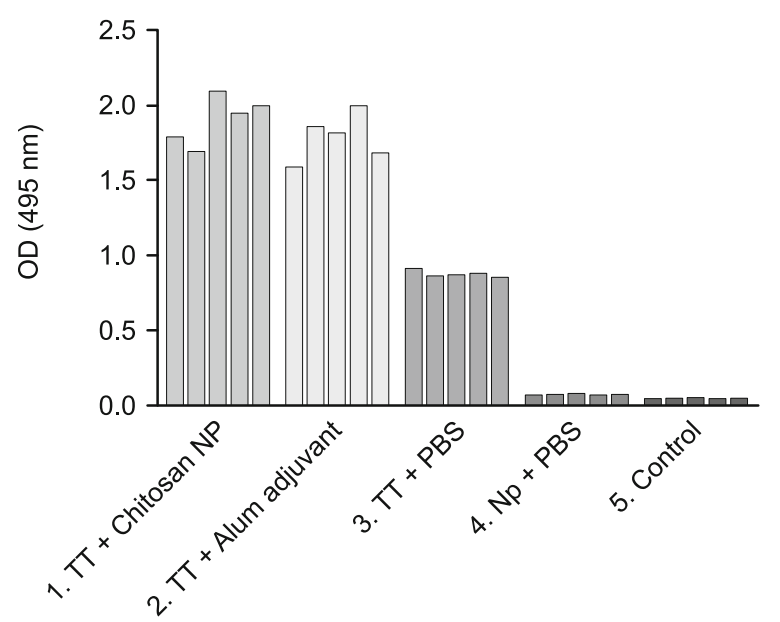

Fig. 3. Tetanus toxoids IgG antibody levels after administration via subcutaneous route.

\section{Discussion}

The present study was aimed at developing tetanus toxoid-loaded chitosan nanoparticles to test immunogenicity characteristics. The purity assessment by SDS-PAGE showed that a single band is located at the top of gel with no extra band. The same result was observed with Zarifpour et al (2013) and Tafaghodi et al with the tetanus toxoid $(8,9)$. No aggregates or fragments were detected, confirming high purity of the purified TT and good immunogenicity. Harsh conditions may cause structure defects and decrease the antigenicity of protein $(10,11)$. The ionic gelation method is a cost-effective method that uses a mild condition to preserve the activity and functionality of reagents $(11,13)$. Siddhapura et al (2016) produced tetanus toxoid-loaded chitosan nanoparticles by using ionic gelation method with average size of $208 \mathrm{~nm}$ and $99 \%$ entrapment. In vivo immunization revealed that microneedle-assisted vaccination induced IgG titer $\sim 1.2$ higher than the current intramuscular tetanus toxoid (12). In the present research, the subcutaneous injection was employed successfully. Sayin et al (2008) investigated the induced immunity of mono-N-carboxymethyl chitosan (MCC) and N-trimethyl chitosan (TMC) nanoparticles through mucosal and subcutaneous routes. The nanoparticle size was found to be in 40-400 $\mathrm{nm}$ range. The present results showed that IgG titers were significantly higher in nano-capsulated TT compared with non-encapsulated TT. Furthermore, MCC induced significantly lower immune responses in mucosal vaccinated group compared with subcutaneously vaccinated group ( $\mathrm{p}<0.01)(13)$. The results of present study showed that the level of antibody was markedly improved in animals vaccinated with TT-loaded chitosan NPs. Mice treated with TT-loaded NPs produced approximately the same level of antibody compared with aluminum hydroxide mice group. In a study, a low dose of influenza vaccine with aluminum adjuvant through intradermal and intramuscular route was compared with normal dose. The result showed that none of the low-dose vaccination strategies is as effective as vaccinating with the normal human dose. However, the low-dose aluminum-adjuvant vaccine is a feasible alternative in case of a limited vaccine supply (14). From the present study, it was evident that TT-loaded NPs were able to induce a strong humoral immune response after administration in mice. Therefore, it can be suggested that this antigen delivery system would be more efficient for enhancing the humoral immune response when compared with traditional adjuvants. Prepared chitosan NPs can presumably decrease the adverse effects while rendering an excellent effect on the humoral immune response. The level of antibody induced by chitosan was eight times higher than with aluminum hydroxide in mice vaccinated with influenza vaccine (10). Chitosan is believed to improve the cellular expansion of lymph nodes in the site of injection, activate macrophage and natural killer cells and prolong retention of antigen in the injection site $(15,16,17)$. Wen et al $(2011)$ investigated the immune response to ovalbumin in mice and showed that ovalbumin-specific $\operatorname{IgG}, \operatorname{IgG} 1, \operatorname{IgG} 2 \mathrm{a}$, and $\operatorname{IgG} 2 \mathrm{~b}$ antibody titers were significantly higher in ovalbumin with chitosan nanoparticles compared with it alone or with chitosan. It was revealed that chitosan nanoparticles have a remarkable potential to increase both cellular and humoral immune responses (16). In conclusion, the results showed that the prepared chitosan nanoparticles (morphology and particle size) are in an appropriate condition. The tetanus toxoid-loaded chitosan nanoparticles of this study could elicit a high humoral immune response. It is suggested that TT-loaded NPs could be a good alternative system for antigen delivery of tetanus toxoid and it seems that chitosan has a great potential as a tetanus vaccine adjuvant and could contribute to the production of novel vaccines for unimmunized individuals.

\section{References}

1. Cook TM, Protheroe RT, Handel JM. Tetanus: a review of the literature. Br J Anaesth 2001; 87: 477-487.

2. Blencowe H, Lawn J, Vandelaer J, Roper M, Cousens S. Tetanus toxoid immunization to reduce mortality from neonatal tetanus. Int J Epidemiol 2010; 39 (Suppl 1): 102-109.

3. Aiyer Harini P, Ashok Kumar HG, Praveen Kumar G, Shivakumar N. An Overview of Immunologic Adjuvants - a Review. J Vaccines Vaccin 2013; 4: 167. doi:10.4172/2157-7560.1000167.

4. Solanki VA, Jain NK, Roy I. Stabilization of tetanus toxoid formulation containing aluminium hydroxide adjuvant against freeze-thawing. Int J Pharm 2011; 29; 414 (1-2): 140-147. doi: 10.1016/j.ijpharm.2011.05.022.

5. Nair LS, Laurencin CT. Biodegradable polymers as biomaterials. Prog Polym Sci 2007; 32: 762-798.

6. Cheung RCF, Ng TB, Wong JH, Chan WY. Chitosan: an update on potential biomedical and pharmaceutical applications. Mar Drugs 2015; 13: 5156-5186. doi: 10.3390/md13085156.

7. Bradford MM. A rapid and sensitive method for the quantitation of microgram quantities of protein utilizing the principle of protein-dye binding. Anal Biochem 1976; 72: 248-254.

8. Zarifpour M, Hadizadeh F, Iman M, Tafaghodi M. Preparation and characterization of trimethyl chitosan nanospheres encapsulated with tetanus toxoid for nasal immunization studies. Pharm Sci 2013; 18: 193-198. 


\section{$71-74$}

9. Tafaghodi M, Jaafari MR, Tabassi SR. Nasal immunization studies by cationic, fusogenic and cationic-fusogenic liposomes encapsulated with tetanus toxoid. Curr Drug Deliv 2008; 5: 108.

10. Bowersock TL, HogenEsch H, Suckow M, Guimond P, Martin S, Borie DL, Torregrosa S, Park H, Park K. Oral vaccination of animals with antigens encapsulated in alginate microspheres. Vaccine 1999; 17: 1804-1811.

11. Tafaghodi M, Sajadi Tabassi SA, Jaafari MR. Induction of systemic and mucosal immune responses by intranasal administration of alginate microspheres encapsulated with tetanus toxoid and CpG-ODN. Int J Pharm 2006; 319: 37-43.

12. Siddhapura K, Harde H, Jain S. Immunostimulatory effect of tetanus toxoid loaded chitosan nanoparticles following microneedles assisted immunization. Nanomedicine 2016; 12: 213-222.
13. Sayın B, Somavarapu S, Li XW, Thanou M, Sesardic D, Alpar HO, Senel S. Mono-N-carboxymethyl chitosan (MCC) and N-trimethyl chitosan (TMC) nanoparticles for non-invasive vaccine delivery. Int $\mathbf{J}$ Pharm 2008; 363: 139-148.

14. Hauge S, Madhun A, Cox RJ, Haaheim LR. Quality and Kinetics of the Antibody Response in Mice after Three Different Low-Dose Influenza Virus Vaccination Strategies. Clin Vaccine Immunol CVI. 2007; 14 (8): 978-983.

15. Zaharoff DA, Rogers CJ, Hance KW, Schlom J, Greiner JW. Chitosan solution enhances both humoral and cell-mediated immune responses to subcutaneous vaccination. Vaccine 2007; 25: 2085-2094.

16. Wen ZS, Xu YL, Zou XT, Xu ZR. Chitosan Nanoparticles Act as an Adjuvant to Promote both Th1 and Th2 Immune Responses Induced by Ovalbumin in Mice. Marine Drugs 2011; 9 (6): 1038-1055.

Received September 2, 2017. Accepted October 30, 2017. 